\title{
UTILIZANDO INSTRUMENTOS PARA AVALIAÇÃO DA PERCEPÇÃO DE DOR EM PRÉ-ESCOLARES FACE A PROCEDIMENTO DOLOROSO
}

\section{USING TOOLS FOR PAIN PERCEPTION ASSESSMENT IN HOSPITALIZED PRE-SCHOOL CHILDREN SUBMITTED TO PAINFUL PROCEDURE.}

\author{
Lisabelle Mariano Rossato* \\ Margareth Angelo**
}

\begin{abstract}
ROSSATO. L.M.: ANGELO. M. Utilizando instrumentos para avaliaço da perepeño de dor em pró-escolares face a procedimento
\end{abstract} doloroso. Rev. Fise. En f.USP. v.33, n.3. p.2:36-49. sot. 1999)

\begin{abstract}
RESUMO
Liste cstudo lewe como objelivos: verificar se a escala de faces de intemsidade de dor possibilita ao pré-escolar identificar sua experiancia dolorosa; idemlificar comporlamentos indicadores de dor "min préescolares submetidos a procedimentos doloroso; conhecer palatras mlilizadas pelo préescolar para descrever a dor em relagäo a procedimentos doloroso. Para tanto foram aplicados trés instrumentos am $1 /$ criansas hospitalizadas submetidas a curativo on coleta de sangue. Os instrumentos nlilizados foram escala de faces, indicadomes comporlamemlais e palauras descriloras de dor. A maioria dos préescolares compreenden e ntilizon correlamente a escala de faces, o que foi evidenciado pelo morimento crescente o (sem dor) a I (dor maxima) na escollha das faces antes a depois do procedimento doloroso. Os comportamentos choro, imóvel e lanzir a testa predominaram como indicalores comportamentais. mostrando-se intensificados na vigencia do procedimento. As verbalizasóes do préseolar relalivas a sua dor revelaram uma tendéncia na utilizagão de termos concretos e de natureza avalialiva on indicativa, resultando cm conceilos variados e pouco precisos.
\end{abstract}

LNITERMOS: Dor. Enfermagem pediátrioa. Pré-oscolar.

\begin{abstract}
The aim of this study was evaluate the ntilizalion of tools for pain perception assessment in th hospitalized preschool children submilled to painful procedures: care of wounds or blood ex/raction. The mlilizaded tools were the faces scale, behavioral indicators and words which describe lhe pain. Most of the preschoolers understood and correctly answered to the faces scale, which can be noticed by the (rescemt movimemt o (no pain) (R) 1 (mavimum pain) during the choice of faces before and after the painful procedure. Crying. immolility and forehead wrikling were the predominant behavioral indicators, inkensified during the procedure. Pre-schoolers verbalizalions abont their pain

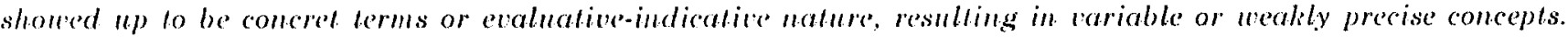

INITERMS: Pain. Pediatric Nursing. Child, preschool.

\section{INTRODUÇÃO}

Apesar da dor ser uma experiôncia humana universal. passou a ser objeto de estudo apenas neste século. entre os anos 50 e 60. Em 1979), a Sociedade Internacional para Estudos da Dor conceituou-a como "uma experiência pereeptual. sensorial e omocional desagradável que está descrita em tormos de lesões teciduais reais ou potenciais. A dor 6 sempre subjetiva e cada inclivíluo apronde a utilizar esto termo através de suas experiencias juvenis e traumáticas..." (IA BARS; WILL.TRR 1993).

As crencas de que a criança não experienciasse dor. além de não possuir capacidade de quantificar lenomenos abstratos, como a intensidade da dor, eram vigentes antes da década de 70 quando o interesse pela pesquisa da dor inlantil teve início (NEDPOR'lll 1995). A partir da decada de 80.

\footnotetext{
* Prolessor Assistente do Departamento de Enfermagem Matemo-Inliantil e Psiquiatriea da Fiscola de Enfermagem da USP.

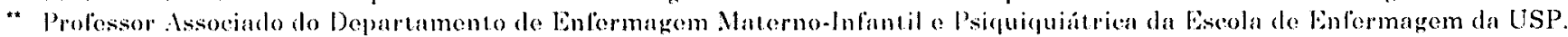


desencadeia-se um considerável aumento do investigações acerea da dor em criança., quando pesquisadores como HESTER (1979). ABU-SAAD (1981) e MeCRATH: CRATG (1989) apresontam evidencias de que crianças sĩo eajazes de indicar os vários niveis de seu sofrimento, caso o adulto lhes proporcione instrumentos adecuados como escalas. diagramas ou desenhos. Estes estudos passaram a constituir elementos (jue possibilitaram uma nova concepcão acerca da dor em crianças.

No inicio das investigaçoes sobro dor SCHULT\% (1971) já advertia que a resposta da criança ao fenômeno pode não ser tão imodiala quanto a do adulo. Sendo assim. para atuar no alivio da dor da criança e preciso primeiro entender eomo ela percebe a clor o depois aprender a antecipar as medidas de alívio ou prevenção da mesma.

Apesar de estudos demonstrarem que erianças tambóm possuem capacidade para experimentar dor. BEYER: WELLS (1989) ressaltam que a avaliação da dor pediatrica o um dos problemas mais desaliantes com que so deparam os provedores de assistência a saúde na infância. Mesmo parecondo claro este fato, muitos profissionais de saúde têm dificuldade em considerar a existencia da dor na crianca lalve\% por ola não se exprossar (la mesma lorma (que os adultos e possuir uma forma peculiar de percebor a experiencia.

AYNSLEY-GRLNN: WARD-PLA'T" (1995). indicando algumas razōes para cxplicar porcue a dor em crianças não recobe a mesma atenção que nos adulios. ressiallam of falo de gue os profissionais habitualmonte não perguntam à criança se cla sente dor motivados talvez pelo desconhecimento de que as erianças monores tem maior dificuldade em eomunicar se está sentindo dor, pela erença de que o comporramento de dor pode ser controlado pela contenção. gue erianças passivamente aceitam o também pola possibilidade dos pais desconhecerem o que está sendo leito com seu fïlho.

Para avaliara dor em eriancas pequenas. eomo pré-escolares. ó necessário enlalizar a sua maneia de perceber a experioncia dolorosal pois a criança desta latia obaria percobo a dor como uma experiencia lísica e convive com ela do uma maneira egocentrical. como mostram om scus csturlos . MBU. S.AD) (1981). BALER: WONG: QUEST (1987). IIURLAY: WIIEIAN (1988) O SIIEIN (1995).

As criancas próescolares. geralmence roagem a uma injeção demonst rando tanta preocupação pela retirada da agulha quanto pela dor roal. Este lato o resultado do pensamento mágico earacteristico da lase pre-escolar que. segundo PlAGli'l' (1967). engtoba as idades entre 2 e 7 anos.

Eim função das limilacoos inerentes ao desenvolvimento da criança. inúmeros estudos têm siclo realizados objetivando identificar recursos que possam ajudar a criança. do pré-escolar ao adolescente. a apresentar informações sobre sua dor. Aguns destes instrumentos, ao longo dos anos, vêm se tornando bastante conhecidos e acessiveis aos prolissionais de saúde, como o instrumento de cores de liland (ELAND, 1981), a escala visual análoga. as escalas numéricas, a escala dos copos, a escala deseritiva do palavras (BAKER: WONG; QUEST, 1987). (WONG: BAKER. 1988), (CLEVE; SAVEDRA, 1993). o a escala das fichas ou Hester's Poker Chip 'l'ool (MACKEYD: JORDAN-MARSH, 1991). Estas escalas demandam certa escolaridade da criança. tanco de leitura de números como de compreensão do conceito cheio e vario.

Dentre as diferentes escalas, a que parece ser mais adecpuala à criança pró-escolar que ainda não se allabetizou ou recebeu conbecimentos aritméticos . a escala das laces que consiste numa técnica em que sois liguras de laces são mostradas à criança indicada por WONG: BALER (1988). Nesta escala. a primeira ligura ó muito sorridente e as expressões vão se transformando, mostrando graus crescentes de tristoya alté chegar à última que é muito triste. Diante da escala a criança escolhe a face que julga mais parecida com a sua própria. em situação de dor.

A partir clesta primeira escala, outras comegaram a ser desenvolvidas. propondo diversos ostilos de laces. Destacamos em nosso meio, a pesquisa roalizada por CLARO (1993), que descenvolveu e validou uma escala de faces para lacilitar a abordagem do tema dor com a criança em idacle escolar. A escala ale laces construida pela autora segue o mesmo princípio de transformação de expressão facial, utilizando-se de figuras familiares à cultura da criança brasileira. Apesar da existencia de instrumentos. pesquisas demonstram guo a dor na infância continua recebendo alenęão limitada e e tratada muito menos intensivamente que a dor no adulto. talvez porque a maioria dos enfermoiros ainda considere que a criança experimenta menos dor que o adulto. conformo observa SCHECH'l'ER (1989) em seu um esturlo. Quanto a submolicação um fenômeno presento na assistência à crianças com dor, esta tem sido motivala pelo temor de hábito e depressão respiratoria e o desconhecimento clos efeitos psicologicos dos opiaceos no alívio da dor om criancas.

Aposar de estudos demonstrarem a possibilidade de carla um dos problemas serem passiveis de controle e prevenção, os mitos permanecem, devido às limilações de conhecimento o troinamento de profissionais em relação ao tema dor. desinformação e confusão entre os conceitos de tolerancia depondôncia lísica psicologica o 
(lepressão respiratória. Estes fatores polem dificultar a comunicação eficaz de ideias corrotas acerea desse assunto, resultando numa avaliacão pouco precisa da dor da criança e no manojo inadequado da mesma.

Ao longo de nossa experiência, e frecpüente ouvirmos dos profissionais que a criança "faz manha" pois o procedimento não causa tanta clor. Como alguém pode decidir por outra pessoa se determinado procedimono causa ou não dor? Quando lombramos gue procedimentos invasivos. sobretudo acjueles em (jue sño utilizaclas agulhas, remetem a criança ao conhecido pavor por estes instrumentos. causa-nos preocupação constatar que o adclulto pode estar sendo incapaz de perceber o solirimento da criança.

$\mathrm{Na}$ maioria dos hospitais é comum observar prescriçoos eomo rotina de um mesmo analgésico para todas as crianças internalas. independentemente de sua queixa. Tal lato leva-nos a indagar se a tolerância a dor cle cada uma delas está sendo efotivamente considerado. bem eomosua (a) acidade de comunicação já cue na maioria das vozes, as prescriçoes são atribuidas a condição "so necessiário"

Dessa forma percebemos que apesar de osludos sobre dor om crianca estarem sendo publicados om diversos países. a pescuisa sobre dor inlantil no Brasil permancee incipienle. Ilojo contamos com alguns autores brasileiros gue estudam o lenômeno dor. entre os quais, destacamos PINEN'l'A (1995) que $1 \mathrm{~cm}$ realizado investigacoes sobre dor om adultos o CONCLICÃO: SII ROBERGE (1985). YALLE: RRANCOSO (1992) O CLARO (199:3) com estudos solore crianças.

Consilleramos urgente e necessário ampliar ostudos o publicaçoes acerea do assunto. no âmbito da Enfermagem. Entendemos que a enformoira encontra-se numa posiço ideal para avaliar a dor da crianca. influenciar o sou controle o ocasionalmente evitas a ocorrencia de experioncias dolorosas desnecessárias. Pode ainda utilizar-se dessa posiça vantajosa para construir a abertum entro a poscpuisa sobre dor o a prática clínicar na tonlativa de dim inuir ou aliviar o sofrimento inlamtil aprendendo a avaliar a criancal com dor utilizander se da grande variedade de abordagens disponiveis. listas porém. não são consideraçoes recentes. llá quase duas décadas. HeSllik (1979) ja restallava a importâneia das enlermeiras desenvolverem uma base solida de conhecimontos sobre avaliacão. plancejamento, intervenção e evolução da criança com dor.

Ao falarmos om assistência efotiva e oficiento. não estamos nos roforindo aponas a realizaça do procedimentos e ulilização do modicamontos elicazes. listamos apontando para outra mancira de olhar a assistencia, um estilo que percorre outro caminho. ou seja. a direção da assistência humanizadia. a cual todos temos direito, sobretudo a criança gue se as veres não consegue com unicarnos sua dor.

Nesse sentido, 6 essencial considerar os dirọitos da criança, indo além do problema de acrediar se a criança pode ou não sentir dor. Isto prossupoos uma rollexão do profissional em perguntar-se: a criança está sentindo dor? qual o impacto (pue a (lor causa om sua vida? (ROSSATO) REKLNI)L. 1996).

A partir desses estudos e indagações e, preocuparlas om contribuir com subsíclios que possibilitem a avaliação da dor aguda da criança préescolar. bom como sua intervenção adequada, decillimos realizar esta posquisa destinada a avaliar a cxperiência de dor aguda na criança pré-escolar. submel idla a proced imento doloroso. A opção pela dor aguda se deveu ao lato de ser esta a natureza de dor mais comum (quo profissionais de saúde enfrentam com crianças hospitalizadas. Quanto à faixa etária pro-escolar. esla foi motivada pela complexidade que ela impóc a avaliação da dor e por existir um menor número de pescquisas envolvendo o pró-escolar.

\section{OBJETIVOS}

- Verificar se a escala de faces de intensidade de dor possibilita a pre-escolar identificar sua experiencia dolorosa:

- Identilicar comportamentos indicadores de dor em pré-escolares submetidos a procedimento (loloroso:

- Conhecer palavras utilizadas pelo pré-escolar para descrever a dor em relação a procedimento doloroso.

\section{METODOLOGIA}

\section{Populaç̃o}

Foi constituida por 11 crianças pré-escolares. eompreendendo o período de 33 a 6 anos de idade, que estavam sendo submeticlas a procedimento doloroso.

A selecano das crianças bem como a entrevista c o preenchimonto dos instrumentos foram realizados pela autora. obedecendo aos seguintes cricóros do soleção:

- Estar na laica chária pre-escolar (3 a 6 anos inclusive): 
- Estar sendo submetida à realização do um procedimento doloroso. que para este estudo foram assim definidos: curativo, coleta de sangue ou retirada de scalp:

- Ser capar de comunicar-se verbalmente e indicar suas necessidades.

\section{Coleta de dados}

A coleta de dados foi realizada em unidades de internação pediátrica de dois hospitais públicos especializados em ortopedia e cardiologia da cidade de São Paulo, no período de agosto de 1995 a março de 1996.

A avaliação das respostas dos pré-escolares à dor aguda foi efetuada por meio da utilização de guatro instrumentos destinados a estimar as dimensões comportamental e pereeptual da dor na eriança .

- Instrumentol (Anexo 1) Caraclerizacão da populacióo. contendo: dados de identificação nomo. idade. sexo): daclos da doenȩa (diagnosstico. data de internação). Os dados de identificação (Anexo 1) foram obticlos através do prontuário da criança.

- Instrumentoll (Anexo 2)-Escala de faces . Neste estudo. instrumento para a coleta de clados de intensidade de dor, foi uma das duas Escalas do Faces de Claro (1993) (Anexo 2), já cue a base desta escala o a identificação de vários níveis de intensidade de dor pela criança. A seleção de apenas uma das escalas, deveu-se a um processo de observação da preferêneia das crianças pela face do personagem utilizada no estudo. A aplicação do instrumento 11 (Anexo 2) aconteceu antes o após o lérmino do procedimento doloroso. quando a criança foi solicitada a apontar na escala. a lace cue mais representava a sua clor. Para tanto, cra explicado a criança que cada face correspondia a uma sensação no momento do proeedimento doloroso. ou seja. a lace () era sem dor. a face 1 uma dor leve, a face 2 uma dor moderada, a face 3 uma dor forte e a lace 4 uma dor insuportável. A seguir era solicitado à eriança que escolhesse a lace mais parecicla com a sua antes e depois do proeedimento doloroso.

- Instrumento 111 (Anexo 3) - Indicadores Comporamentais: o instrumento utilizado neste ostudo foi adaptado a partir do estudo (lo ABU -SAAD (1984). Os Indicadores Comportamentais constituiram-se de uma lista de comportamentos que incluem vocalização (grito, suspiro, choro. gemido, soluço). expressão lacial (abrir a boca. fochar os olhos. cerrar os dentes. arregalar os olhos, apertar os labios. franzir a testa) e movimentos corporais (imovel. agitado. protegido, encolhido. tenso). Para a aplicação do instrumento III (Anexo 3) observamos os comportamentos da criança de acordo com os Indicadores. antes do procedimento doloroso, clurante a realizacião do mesmo e depois de finalizado, registrando todos os comportamentos apresentados.

- Instrumento IV (Anexo 4) - Palawras Descritoras Este instrumento constava de uma questão norteadora que possibilitou a identificação de palavras mais utilizadas pelos pré-escolares na deserição de sua dor. () instrumento IV (Anexo 4) foi aplicado aj) os o término do procedimento doloroso. quanclo foi formulada a soguinte questão à criança: "Como o a sua dor?". A s respostas eram a seguir registradas utilizando-se as próprias palavras empregadas pela criança para a resposta.

\section{APRESENTAÇÃO E ANÁLISE DOS RESULTADOS}

Os resultados foram organizados em gráficos e tabelas com pereentagem e número absoluto sendo a) licarlos testes estatísticos aos resultados da escala de faces, julgados necessários segundo orientação estatistica. com o objetivo de sumarizar as informaçoes reforentes aos "scores" obtidos para o Instrumento II, seguindo recomendação de SIEGEL (1975). Foram calculadas as médias e desvios-padrão de cada um dos grupos por sexo, idade e seus cru\%amentos. Deve-se notar que estas medidas são de caracter exclusivamente descritivo, já que para os testes foram utilizados procedimentos não paramotricos, mais adequados para a mensuração ulilimadia ("scores").

Os Indicadores Comportamentais foram analisados segundo porcentagem e número absoluto. c organizados em cualros de modo a evidenciar respectivamente, os comportamentos apresentados polas crianças antes. clurante e apos os procedimentos. As palavras descritoras de dor foram analisalas em função dos significados que elas continham.

Foram aplicados os instrumentos em 44 crianças, sendo que 3 delas foram excluílas da amostra por não terem conseguiclo apontar na escala de faces a que mais representava a sua dor percebida antes do procedimento. Em virtude destas exclusões, a população total constituiu-se de 41 crianças.

\section{Caracterização da população}

Na população estudada houve o predomínio do sexo masculino $28(68.29 \%)$ e la faixa etária entre 5 - (i) anos $26(63,41 \%)$ como podemos observar na 'rabela 1: 
Tabela 1-Distribuição das crianças de 3 - 6 anos sogundo o soxo o a laixa otária. São Paulo. 1996.

\begin{tabular}{lcccccc}
\hline & \multicolumn{2}{c}{ Masculino } & \multicolumn{2}{c}{ Feninino } & \multicolumn{3}{c}{ Total } \\
Idade & $\mathrm{N}$ & $\%$ & $\mathrm{~N}$ & $\%$ & $\mathrm{~N}$ & $\%$ \\
\hline $3-4$ & 12 & 29.27 & 3 & 7.32 & 15 & 36,59 \\
5.6 & 16 & 39.02 & 10 & 24.39 & 26 & 63.41 \\
\hline TOTAL & 28 & 68.29 & 13 & 31.71 & 11 & 100 \\
\hline
\end{tabular}

Tabela 2 - Distribuição das crianças de :3 - (; anos segundo diagnóstico. São Paulo. 1996.

\begin{tabular}{|c|c|c|}
\hline \multirow{2}{*}{$\frac{\text { Idade }}{\text { Diagnóstico }}$} & \multicolumn{2}{|c|}{$3-6$ anos } \\
\hline & $\mathrm{N}$ & $\%$ \\
\hline Fratura suppacondiliana de úmero & 19 & 16,34 \\
\hline Fratura de rêmur & 4 & 9.76 \\
\hline Fialura dos ossos da perna & 2 & 4.88 \\
\hline Ferimento de mão & 1 & 2,44 \\
\hline lecrimento de perna & 1 & 2,44 \\
\hline Ferimonto de pé & :3 & 7.32 \\
\hline Correç̃o de deformiclacle de MID & 1 & 2,44 \\
\hline Correç̃o de MSD (anoxia de parto) & 1 & 2,44 \\
\hline Seqüela de pioartrite de quadril & 1 & 2,14 \\
\hline Artrite roumatoide juvenil & 1 & 2.44 \\
\hline Psoudoartrose & 1 & 2.11 \\
\hline l uxaç̃ono congênita de quadril (LCQ) & 2 & 1,88 \\
\hline Insufieiência cardiaca congestiva (ICC) & 1 & 2.44 \\
\hline Comunicação interventricular (CIV) & 1 & 2.11 \\
\hline Comunicação interatrial (Cl: & 2 & 1.88 \\
\hline TO'TAL & 41 & 100 \\
\hline
\end{tabular}

A alta incidência de afocções. determinadas por acidentes como causa de internação da população estudala of o que mais chama a atenção. haja visto a ocorrêneia de $30(73.18 \%)$ acidentes. dos cuats 25 $(60.98)$ resultaram em lraturas. Observamos na 'I'LBESLA 2 que a lratura supracondiliana de úmero apareceu om maior númoro 19 (46.34\%). Segundo ALBBRTONI: MA'TSUMO'TO (1993), esta 6 a mais frecüence fralura do eotovelo em eriancals e corresponde a aproximadamente 60 a $70 \%$ das lesoces desta articulacão. Ocorre gemalmente entro os 3 a 10) anos de idade. ab)rangendo mais as crianças entre 5) a 8 anos. lendo maior provalóncia no soxo masculino.

Cl.ARO (1993) comenta que os índices de dor em membros superiores e inferiores de escolaress encontrados em seu estudo. apresentam um emponente que liga as atividades das crianças a acidentes, como quedas de bicicletas, quedas ao correr e pancadas, oeasionando ferimentos, fraturas ou traumatismos.
A provalência do scoxo masculino na população estudada deve-se possivolmente, ao tato dos meninos preferirem atividades lúdicas mais arriscadas do que as meninas. ocasionando maior número de fraturas e ferimentos. A mesma interpretação pode ser aplicada a faica etária en tre $5-6$ anos a qual corresponde a maior autonomia da criança em anclar o brincar sozinha, acarretando acidentes com maior freqüencia.

Sogundo \%UCKERMAN: DUBY (1985). o mundo da criança pré-escolar deixa de estar ligado apenas ao sistema sensitivo-motor clevando seu conhecimento para niveis mais clevados e baseados no uso de símbolos. sendo classicamente conhecido como pensamento pre-operacional. Nesse estágio, o pensamenco pró-lógico da criança indica que ela não compreende folalmente as relações de causa e efeito e não pode generalizar a partir de experiencias concretas. São ostas características ilógicas e egocêntricas do pensamento gue podem acarretar acidentes. acrescentam \%UCKERMAN; DUBY (1985). 
As inlernações motivadas por correcões de deformidades e malformaçōes também con lïguraram como aspecto caractoristico da população estudada. Segundo ALMEIDA (1996). a maioria das correções cirúrgicas cardíacas principalmente as definitivas, ocorre na laixa etária do pré-escolar, pois permite o fechamento espontâneo de algumas comunicações intercavitárias menores.

Os procedimentos dolorosos vivenciados pelas crianças deste estudo são mostrados na TABELA 3:

Tabela 3 - Distribuiço das crianças de $3-6$ anos segundo procedimentos dolorosos realizados. São Parulo. 1996.

\section{$3-6$ anos}

\begin{tabular}{lcc} 
Procedimento doloroso & N & $\%$ \\
\hline Curativo & 21 & 58,54 \\
Coleta de sangue & 15 & 36,58 \\
Retiradla de scalp) & 2 & 4,88 \\
\hline TOTAL & 41 & 100 \\
\hline
\end{tabular}

O curativo foi o procedimento mais observado 21 (58.54\%). por decorrência do grande númoro de fraturas como motivo da internacão, determinando correçoses cirúrgicas e consegüente realização de curatives. A coleta de sangue foi outro procedimento observado $15(36,58 \%)$ como rotina da infernaciano para cirurgia. Segundo ELAND (1981), as crianças possuem sentimentos negallivos em relação a injecooses pois vivenciam. Treqüentemente, estas oxperioncias associalas a dor durante a hospitalizaçõo. WONG: BAKliR (1988) comentam (jue punções venosas, picadas nos dedos e injeções sio procedimentos comuns durante a in ternação das crianças. e são consideradas intervenções dolorosas polas crianças.

\section{Escolha de Faces Antes e Depois do Procedimento Doloroso}

$A$ guantificação da experiência dolorosa foi realizarla antes e dejois do procedimento doloroso. a partir da aplicação da liscala de Faces.

Tabela 4 - Distribuição de crianças de 3-6 anos de acordo com a lace escolhida antes e depois do procedimento doloroso. São Paulo, 1996.

\begin{tabular}{lcccc}
\hline \multirow{2}{*}{ Valores atribuidos às faces } & \multicolumn{2}{c}{ Antes } & \multicolumn{2}{c}{ Depois } \\
\hline 0 & $\mathrm{~N}$ & $\%$ & $\mathrm{~N}$ & $\%$ \\
\hline & 14 & 34.15 & 4 & 9,76 \\
2 & 14 & 34.15 & 10 & 24.39 \\
3 & 6 & 14.63 & 12 & 29,95 \\
4 & 2 & 4.88 & 10 & 12,19 \\
& 5 & 12.19 & & 4,39 \\
\hline TOTAL & & & 11 & 100 \\
\hline
\end{tabular}

Os resulados da l'ABlict 4 permiliram conhecer o comportamento goral da população estudada. diante da Escala de Faces, antes e depois do procedimento doloroso. Podemos observar que a maioria das crianças 28 (68.30\%) escolheu as faces 0 o 1 antes do procedimento doloroso o que depois do mesmo, maior número de erianças 12 (29.27\%) optou pela face 2. Atribuindo-se as laces valores de 0 (som dor) a 4 (dor máxima). pôle-se obter a média de intensidade de dor gue as crianças expressaram antes e depois do procedimonto doloroso: média antes
$=1.30$ modia clepois $=2.2$. Isto mostra que as crianças utilizaram as faces que indicam mais solimento para explicar sua experiência após o procedimento doloroso.

Estes resultados significam que as crianças estavam diante de uma experiência dolorosa, é indicaram acuradamente. CLARO (1993) mostra, em seu estudo, um alto indice de movimento crescente $0 \Rightarrow 1$, ou scja, um maior número de crianças na faixa etária escolar optando pela face 0 ao indicarem ausência de dor o um maior número de crianças 
escolhendo as faces 3 e 4 para indicar dor. No mesmo 1. Wabalho. ao serem guestionalas a respeito do como liearam apos alguma estratégia de alivio da dor. as crianças apontaram para as figuras de menor valor na escala.

Detalhando um pouco mais a análise dos rosultados, construímos o QUADRO 6, onde se pode observar. que das 11 crianças deste estudo, $7(17,07$ $\%)$ optaram polo movimento (lecrescente $(0 \neg 4)$. Neste estudo denominamos movimento decrescente a escolha por uma face de maior valor leita pelo pré. escolar depois do proeedimento doloroso .

Quadro 6 - Distribuição de crianças segundo sexo. laixa etária e oldão pelo movimento decrescente $(0 \Leftarrow 1)$ clurante a escolha das faces. São Paulo. 1996.

\begin{tabular}{lcccc}
\hline Criança (10) & Sexo & Idade & Antes & Depois \\
\hline 27 & M & 4 & 3 & 2 \\
36 & $M$ & 1 & 1 & 1 \\
11 & $M$ & 5 & 1 & 0 \\
12 & $M$ & 6 & 1 & 0 \\
35 & $M$ & 6 & 2 & 1 \\
5 & $F$ & 5 & 1 & 3 \\
9 & $F$ & 6 & 1 & 2 \\
\hline
\end{tabular}

Chamou-nos a atenção tal resultado, porém não consideramos que signilica que as crianças não tenham entendido a Esscala. já que elas espontanoamente. explicaram o motivo da escolha da face. Durante a coleta de dados deste estudo. notamos diversos acontecimentos, antes do procedimento doloroso. (jue podem ter influenciaclo estas respostas "decrescentes". confirmando a percepção do entendimento da escala de faces pelas crianças.

A opção por um valor alto da escala do faces antes do procedimento doloroso, muitas vezes. deveuse ao lato da criança já estar sentinclo dor antes do procedimento doloroso (curativo ou coleta de sangue) o esto atuar apenas como um agravante do sintoma álgico. Algumas crianças demonstravam reaçoos de medo a simples aproximacão do prolissional.

\section{."Tí doendo, não aperla, lio!"}

Outras ao vorem o carrinho de curativo já comecavam a gritar:

- Hi, é ruim, eu não goslo de fazer curalico."

"Vai me corlar."
Agumas crianças ao verem o técnico do laboratorio já começavam a chorar e verbalizavam seu modo pola agulha.

- "Não quero tomar injeção."

Não podemos deixar de destacar, também, o relato das eriancas em que o medo permeava as siluacoes, fazendo com que elas escolhessem as faces de maior valor. explicitando assim tambem seu sentimento em relação ao procedimento.

"Minha dor nôro passa, tenho dor e medo junlo no carralico."

-"Tenho medo que dói".

E preciso destacar que a maioria destas criancas explicou que antes do procedimento doloroso guase ná sentia dor. listes resultados evidenciam a presença do medo compondo a experiência dolorosa.

Nos resultados observamos ainda que 6 (14.63 \%) criancas desto estudo mantiveram inalterada sua escolha de laces antes o depois do procedimento (loloroso. como mostra o QUADRO 7.

Quadro 7 - Distribuição de crianças segundo sexo. faixa etária e opção inalterada na escolha das faces. Sĩo Paulo. 1996.

\begin{tabular}{lcccc}
\hline Criança (n") & Sexo & Idade & Antes & Depois \\
\hline 14 & $M 1$ & 3 & 0 & 0 \\
26 & $N 1$ & 4 & 1 & 4 \\
40 & $N 1$ & 1 & 0 & 0 \\
21 & $M 1$ & 5 & 2 & 2 \\
$2: 3$ & $M 1$ & 6 & 1 & 1 \\
11 & $M 1$ & 6 & 1 & 1 \\
\hline
\end{tabular}


As cvidências de que clispomos são insulicientes para lazermos interprotacooses procisas destes comportamento. É possivel que estas crianças não tenham compreendido a Escala, como também é possivel que elas estivessem num nivel de ansiedacle tal. que as impedisse do prestar atenção a qualquer solicitação externa. Ainda assim. temos uma lendêneia a acreditar que estas crianças poderiam estar com medo. e por isso sequer consideraram a solicilação para a utilização da escala.

\section{Indicadores Comportamentais}

Os comportamentos indicadores de dor manifestados pelas crianças entre $3-4 ; 5.6$ anos desto estudo estão apresentados nos QUADROS 8 e 9 para facilitar a visualização dos comportamentos por laixal etária.

Quadro 8 - Distribuiç̃o de crianças de 3.4 anos $(n=15)$ do acordo com os comportamentos indicadores de (lor. São Paulo, 1996.*

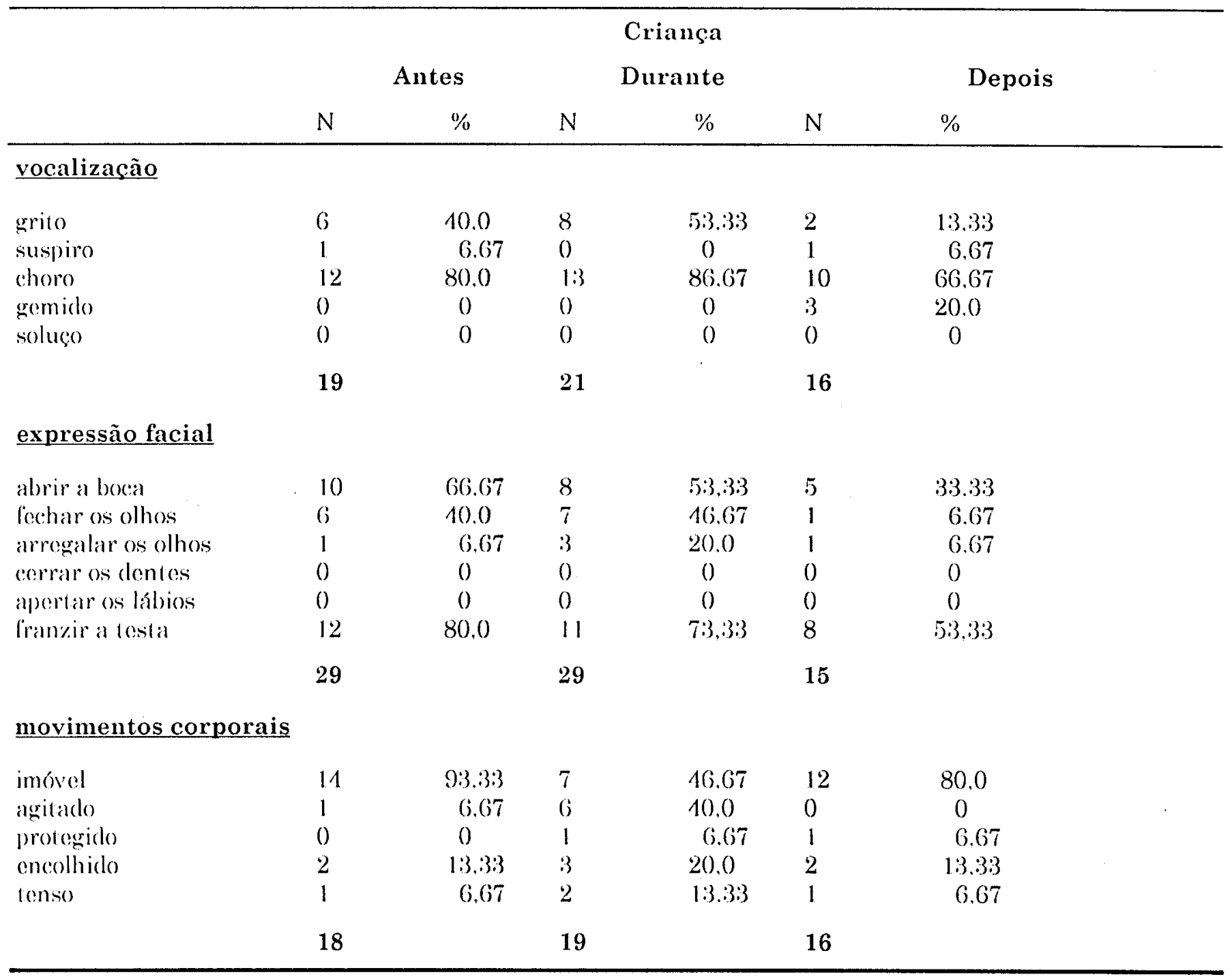

* as criancas apresentaram mais de tum comportamento. 
Quadro 9 - Distribuição de crianças de 5-(3 anos (n=26) do acordo com os comportamentos indicadores de dor. São Paulo. 1996.

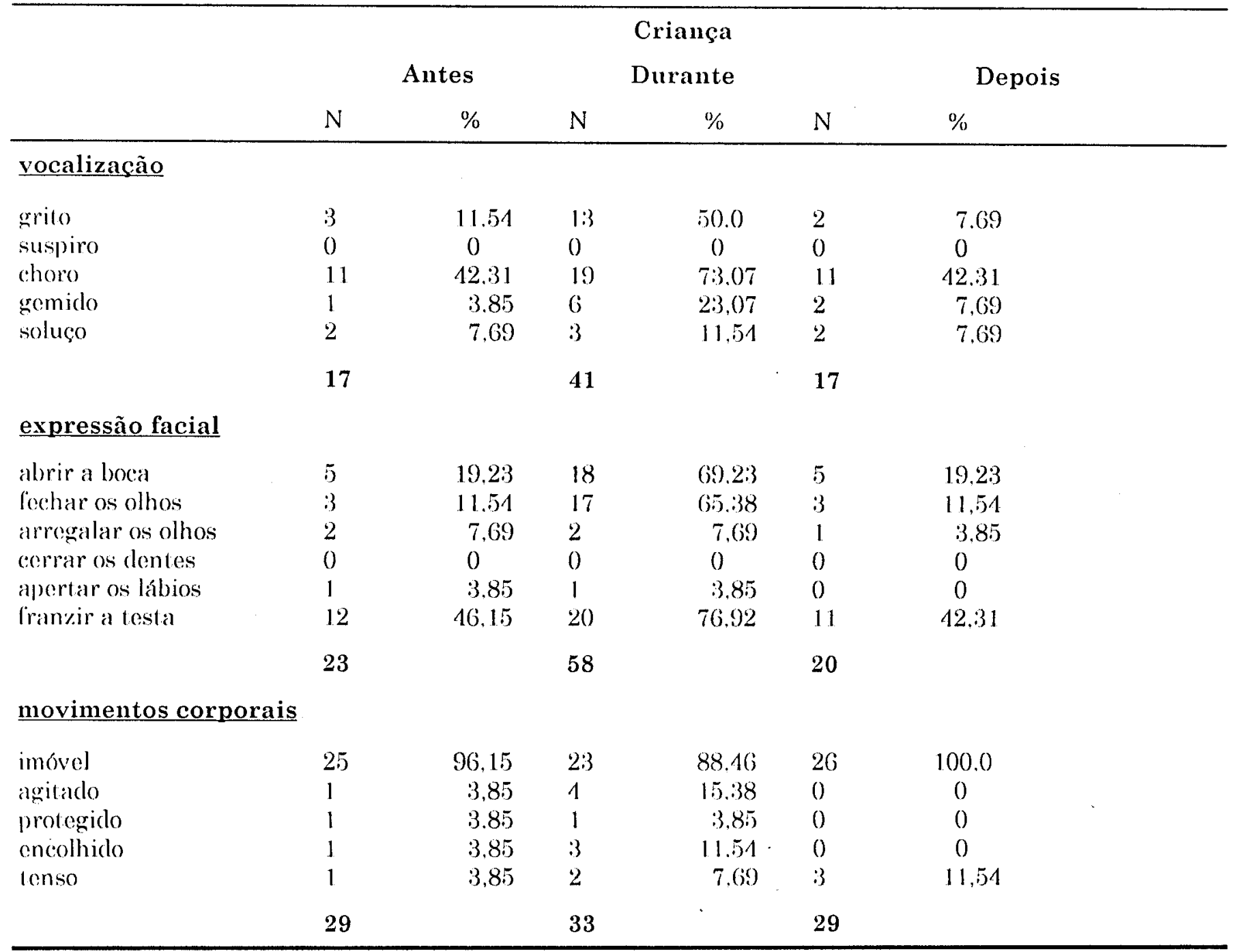

A análise dos resultados mostra uma resposta geral e difusa. ainda que o predomínio seja "choro" anles, durante e depois do procedimento cloloroso. seguido pelo "grito". Analisando os QUADROS 8 e 9 , observamos que, de todos os indicadores do instrumento. apenas "cerrar os dentes" não foi obsorvado para os dois grupos de idade (3-4: 5-6 anos). 'Todos os demais foram utilizados pela crianca para indicar dor. Nolamos o predomínio dos comportamentos choro (vocalizaçao). franzir a testa (expressão lacial) e imóvel (movimentos corporais) durante o procedimento doloroso. Observamos que a vocalização "soluço". a expressão facial "cerrar os dentes" e "apertar os lábios" não foram ap resentadas pelas crianças entre $3-4$ anos em nenhum momento (lo procedimento doloroso, como mositramo QUADRO 8. Do mesmo modo, as crianças entre 5.6 anos não apresentaram a vocalização "suspiro" e a expressão facial "cerrar os dentes", também om nenhum momento do procedimento doloroso, como podemos observar no QUADRO 9. A expressão lacial
"Tranzir a testa" prevaleceu, antes, durante e depois do procerlimento cloloroso seguida pela expressão "abrir " boca". Nos movimentos corporais, o comportamento "imovel" alparece predominante antes, durante e depois (lo procedimento. muito provavelmente, devido a tensão e ao medo que a criança vivencia nestas situações. Lsta percepção encontra outra evidência na ausência do comportamento "agitado" depois do proceclimento doloroso, levando-nos a acreditar que tenha ocorrido pelo fato da criança sentir-se aliviada por ter acabado o procedimento, passando a ficar mais relisiala.

Os resultados deste estudo vêm corroborar os achades da pescuisa realizada por ABU-SAAD (1984) com crianças da laixa etária de 9.15 anos, na qual aparecem, lambem com maior lreqüencia, os comportamontos franzir a testa (expressão facial) e imovel (movimentos corporais). Alem disso, é possivel (que a ausência do choro (vocalização) no estudo acima, deveu-se ao fato do escolar e do aclolescente não manifestarem este comportamento 
por vergonha. fato característico destas faixas etárias. o quo não acontece com o pré-escolar.

BEYER: WELLS (1989), STLIENS: JOHNSTOON (1992), ECOFFEY: DESPARMEN'T (1993) tambem ressaltam que os aspectos comportamentais que a criança com dor apresenta são a expressão lacial, o choro o o movimento do corpos. Enliatizam ainda que esturlos que utilizam várias abordagens para a avaliaçiono da dor infantil indicam que a expressão lacial e a resposta mais eonsistente. Entretanto. não há uma expressão facial precisa associada à dor. As açoes laciais que ma is contam para diferenciar a dor de outras condições são: sobrancelha saliente. olhos fechados comprimidos, bocal aberla, lingua tensa e intensilicação do suko nascorabial.

ECOPREY: DESP.ARME' (1993) oxplicam a razão dos comportamentos inclicando que a dor produz uma angústia, uma agitação incontrolada na criança com o risco da rotirada involuntária de curalivos e drenos.
HES'TER (1995) pressupõe que seja possível que os comportamentos representem a intensidade da dor ou que sejam a forma da criança preveni-la ou entrentála.

O comportamento 6 um indicador presente na oxpressão da dor pela criança ou pelo menos do desconforto o ovidente que os resultados deste estudo confirmam a existoncia de dor ou desconforto.

\section{Verbalizações das crianças}

Da população estudada, 14 crianças responderam à questão formulada, oferecendo algumas frases como respostas, e 8 crianças responderam "não sci” à questão.

Considerando-se que o objetivo do estudo era conhecer as palavras utilizadas pelo pré-escolar na descrição ou indicação do sua dor, as respostas serão apresentalas conforme a ocorrência por grupo de iclade. como mostra o QUADRO 10.

Quadro 10 - Palavras descritoras de dor segundo crianças entro 3 e 6 anos. São Paulo, 1996.

\begin{tabular}{|c|c|c|}
\hline Idade (anos) & Resposta & Classificação* \\
\hline $3-4$ & "I)oi a dor do nenê" & figura de linguagem \\
\hline $3-4$ & "F́ docki" & figura de linguagem \\
\hline $3-4$ & "Dói bastante" & cognitiva-avaliativa \\
\hline $3-4$ & "É uma dor que doi" & figura do linguagem \\
\hline $5-6$ & "É (pucloradar a perna" & inclicativa \\
\hline $5-6$ & "Dói pouco" & cogniliva-avaliativa \\
\hline $5-6$ & "Um pouco dócla. polo jeito tá muito quente" & sensorial-rliscriminativa \\
\hline $5-6$ & "Não docu" & cognitiva-avaliativa \\
\hline $5 \cdot 6$ & "Dói acpui" & inclicativa \\
\hline $5 \cdot 6$ & "Não dói nadla" & cognitiva-avaliativa \\
\hline $5 \cdot 6$ & "Dói muito. lé ruim" & cognitiva-avaliativa \\
\hline $5-6$ & "Saju do lugarar o meu braço" & indicativa \\
\hline $5-6$ & "Os lerros" & indicativa \\
\hline $5-6$ & "l'ava doendo muito" & cognitiva-avaliativa \\
\hline
\end{tabular}

* adapuado de MIELZACR (1975)

Tontando compreender as respostas obtidas. as mosmas foram analisadas frente a classificação de NEJ\%ACK (1975) para descrilores de dor (sensorial-discriminativa. motivacional-aletiva e a cognitiva-avaliativa). Pode-se direr que 6 respostas do estudo possuem um conteádo connitivo. avalialivo, indicalo pelo uso de palavras que determinam um valor ou grau de dor (bastante. pouco. nada. muito). A classilicação sensorial. discriminativa aparece como complemento de uma resposta. na qual a crianca emprega a palavra quente. para referir-se a uma sensaç̃o térmica. A classificasão motivacional-afoliva indicala por MELZACK (1975) nio aparece nas respostas.
No entanto. surgem outras duas classes de respostas, não abordadas por MELZACl (1975), uma (lo natureza inclicativa (4 respostas), quando a criancia aponta a causa ou local da dor (é quebrada a perna doi aqui), e outra (juando a criança utilizase do pleonasmo como resposta (dói a dor, é uma dor (que (loi).

Nestes casos. tanto o uso desta figura de lingurigem como da resposta indicativa, podem sugerir o desconhecimento destas crianças do senticlo da palavra (lor. 1 mesma in terpretação pode ser aplicacla às 8 crianças que responderam "não sei" quando foram solicitadas a verbalizar sobre como cra sua dor o as 19 crianças do estudo não responderam à questão do Anexo 4. Estas respostas 
confirmam resultados de outros estudos relativos à compreensão que a criança tem da dor.

HURLEY: WHELAN (1988) afirmam que a criança, no estágio pré-operacional, compreonde a dor como algo que a liaz licar doente, ou aincla. alguma coisa que fere seu corpo. 'Tan to S'TEIN (1995) como PRICE (1990) referem-se a habilidade de crianças do 3 ou mais anos de idade para identilicar a localização de sua dor. bem como a capacidade na utilizaç̃o de palavras descritivas de sua dor, ressaltando que o préescolar apresenta maior facilidade para identificar a localização da dor. Segundo HESTER (1995). as crianças pré-escolares utilizam -se de termos mais concretos para identificar a dor.

Comparando nossos resultados com outros estudos. encontramos achados semelhantes em ABU-SAAD (1984). que estudando erianças de 9 a 15 anos de idade. identificou. nesta latixa etária. a utilização de 17 palavras sensoriais-discriminativas e 1 cognitiva-avaliativa para descrição da dor e em JERRET': EVANS (1986) identificaram que crianças de 5 a 6 anos de idade utilizaram palavras sensoriaisdiscriminativas com mais frecüencia do que motivacionais-aletivas ou cognitiva-avaliativas.

Estes resultados, juntamente com os do nosso estudo. são evidêneias de que os relatos verbais de crianças pequenas não são acurados o que podem variar consideravelmente. Apesar desta variaç̃o o. da pouca precisão encontrada nas respostas, não podemos concluir acerea de imaturidade cognitiva destas criancias como tradicionalmente ocorre quando so toma por base a descrição do seu estágio de pensamento e a crença de que os concoitos são produto basicamente da estrutura cognitiva.

Hoje. novos paradigmas de analise dos comportamento da criança estäo senclo propostos. YOOS (1994) em seu estudo, apresenta argumentos resultantes de pesquisa na área do inteligencia humana. indicando que são as mudanças nos conteúdos (e não em estruturas cognitivas) quo levam a pessoa a niveis mais claborados de pensamentos. Nesse sentido. talvez o conhecimento demonstrado pela criança acerca da dor noste estudo. reflita seu pouco acesso a informaçós relevantes sobre sua dor. muito mais do que sua imaturiclacle cognitiva.

\section{CONCLUSÕES}

Os resultados deste estudo sobre a utilização (le instrumentos para avaliação da pereepeño de dor om pro-escolares face a procedimento doloroso. realizado com 11 crianças submetidas a curativo ou coleta de sangue. permitiram concluir que:
- Das 41 criancas do estudo, 28 (68,29\%) tiveram uma tenclência a escolher faces à direita na escala, resultando num movimento na escolha das laces. antes e depois do procedimento doloroso. A maioria das crianças escolheu faces que indicam maior sofrimento após o procedimento doloroso. Nesse sentido, pode-se concluir que estes pré. escolares compreenderam o utilizaram corretamente a escala de laces na identificaça da dor que experienciavam.

- A população estudada exibiu um tijo de resposta comportamental geral o difusa. Ainda assim. houve um predomínio da categoria choro como vocalização, da categoria imóvel como movimonto corporal e da categoria franzir a testa, como expressão lacial, nos trôs momentos (antes, durante e depois do proceclimento). Verificou-se no entanto. que tais categoria cram intensificadas bem como os demais. comportamentos durante o procedimento.

- Quando solicitados a descrever verbalmente a dor que experienciam, as respostas forneciclas por 11 pré-escolares do estudo, permitiram identificar a utilização de contoúdo cognitivo-avaliativo. de conteúdo do natureza indicativa e também a utilizaça do ligura do linguagem (pleonasmo) na expressão de seu conceito solore dor. A tendência do pró-escolar em utilizar um conteúdo no cjual termos concretos e disponiveis para explicar a dor estão prosentes, resultou portanto om conceitos variados e pouco procisos.

\section{CONSIDERAÇÕES FINAIS}

A realização desta investigação desencadeou muitas reflexóes, (que ultrapassam o fenômeno dor. mas que estão a ele associadas. A começar pela crianca. sobre a maneira como a vemos. Relendo KORC\%AK (1986), foi inevilavel associar alguns de seus pensamentos as crianças deste trabalho, pela capaciclade (jue este autor tem de captar de maneira p) recisa a percepção da criança como um ser pequeno:

"Todos nós crescemos convencidos de que o grande vale mais do que o pequeno...É chato esticar-se lodo na ponla dos dedos e não conseguir alcancar o objelo almejado. .... criansa ja sabe que não há como resistir... A sensacão de impolência faz surgir o cullo pela forsa... É alravés do nosso exemplo que a crianca aprende a menosprezar aquilo que é fraco".

Diante dessas palavras. 6 possivel pensar que diante da dor, a eriança não pode nada, nós os 
profissionais podemos tudo. Quem é capaz de dizer se a criança sofre em segredo? Afinal, as dúvidas e incertezas infantis nos parecem tão desprovidas de seriedacle...

Será que estamos deixando a criança sentir dor por desconsiderarmos sua forma de expressá. la? Será que o problema da criança ser menos medicada que o adulto está em sua limitada capacidade de comunicar sua dor?

Quantas vezes não desconfiamos da criança. não a levamos a sério. tratamo-la com má vontade. não acreditamos quando diz sentir medo ou dor.

Apesar de nossa desatenção, a criança vivencia a medos, às vezes exteriorizando-os, outras guardando-os para si. Muitas vezes seus esforcos em enfrentá-los fracassam ou são contrariados pelos adultos, e como conseqüência, a criança of muitas vezes colocada desprotegida, em confronto com situações que ultrapassam suas capacidades de defesa e de compreensão

A hospitalização e tudo o que cerca a criança, incluindo os inúmeros procedimentos dolorosos pelos quais ela deve passar é uma das situações que a criança passa. e constatamos que não é preparada para esta situação, nem para os procedimentos hospitalares. A criança muitas vezes, associa a morte à violência, podendo acrẹditar que procedimentos que cortam ou furam alguma parte do corpo significue morrer. (ANGELO), 1986).

Nesse sentido, apos aplicarmos os instrumentos deste estudo, e interagirmos com seus resultados. não podemos ficar incliferentes ao lato de que a criança pode e deve ser vista como uma pessoa que possui inteligência, vontade e uma historia pessoal, que necessita de recursos para avaliar a sua dor de molo mais abrangente do que os que avaliam apenas a intensidade dolorosa. instrumentos que permitam à ela expressar molhor a sua percepção da dor. Recursos que consigam atingir talvez sua experiência global de dor.

Polos resultados, vimos que a criança reage com emocões. Consideramos que atribuir o comportamento ou reação de dor da criança a emoções, como normalmente ocorre (medo, ansiedade, raiva) não deve ser interpretarlo como algo menor na experiência da criança e. portanto. menor quanto à consideração do profissional. Ao contrário. deve ser diagnosticado como solrimento passivel de intervenção.

Não é difícil perceber que a criança se eneontra diante de algum sofrimento, seja ele de ordem lísica ou emocional. Ela pode não conseguir expressar-se verbalmente ou descrever de maneira acurada sua dor, mas ela é capaz de expressar sua experiência no olhar, no rosto, no corpo, na voz, ainda que estes sejam um grito, um comportamento agressivo, um olhar arregalado, uma tentativa de fuga. A criança, efotivamente, possui limitações que tornam a comprcensão de suas experiências às vezes complexa, porque exigem do adulto (pais ou profissionais) um esforço, não para descer até ela como tantos pensam, mas para subir ate ela como diz KORCZAli (1986). Será que estamos diante de uma resistência em chegar até a criança?

Isto nos leva a reflexões também sobre o profissional que interage com a criança, sobretudo o onfermeiro. O que será que determina o comportamento do enfermeiro na avaliação da dor da criança? Como se sente em relação ao preparo para esta avaliação? Que crenças e que tipo de julgamentos utiliza nesta situação?

Quando refletimos sobre estas questões, compreendemos o quanto pode ser complexo o processo de avaliação da dor sobretudo se levarmos em consideração o quanto pode ser difícil para o profissional clissociar a imagem da criança idealizada e organizada socialmente pelo adulto, daquela que ela realmente é ou pode ser. (ANGELO, 1993)

$O$ esforço para compreender a experiência da dor parece pertencer aos clois lados: o lado da criança que tem a tarefa de tentar comunicar algo, e para a qual não está muitas vezes plenamente instrumentalizada (do ponto de vista de domínio cognitivo e de informações) e o lado do enfermeiro. que para aliviar o sofrimento da criança tem a tarefa de desvendar o significado de suas manifestaçōes e fornecer-lhe novos fatos que atuarão como recurso para a roorganização do con hecimento que a criança possui da situação.

Realizar um esforço quando não se conhece os caminhos não é tarefa fácil, tanto para a criança como para o enfermeiro. f́. preciso apoiar a ambos nesto esforço necessário: a criança para ter condição de nos falar sobre sua dor, e o enfermeiro para ser capaz de dar a voz a criança e de ouvi-la.

\section{REFERÊNCIAS BIBLIOGRÁFICAS}

ABU.SAAD. H. The assessment of pain in ehildren. Issues Compr. Pediatr. Nurs., v. 5. n. 5-6. p. 327-35. 1981.

ABU.SAAD, H. Assessing children's responses to pain. Pain., v 19. p. 163.71, 1984.

AL.BIERTONI, W.M: MATSUMOTO. M.H. Fratura supracondiliana do úmero. In: BRUSCHINI. S. Ortopedia pediatrica: a ortopedia do nascimento ao inicio da marcha, a ortopedia do início da marcha à adolescencia, fraturas e lesóes traumáticas mais freqtientes. Sno Paulo. Atheneu, 1993. cap.56, p. 262.4.

ALMEIDA. F.A. O brinquedo terapêutico como intervenção de enfermagem para a crianga submetida à cirurgia cardiaca na unidade de recuperação pós-operatória. São Paulo, 1996. 115p. Dissertação (Mestrado) - Escola de Enfermagem. Universidade de São Paulo. 
ANGELO. M. Hospitalização: Uma experiéncia ameaçadora. In: STEINER. M.H.F. Quando a crianca não tem vez: violencia e desamor. São Paulo. Pioneira. 1986. cap.11, p.101-8.

ANGELO. M. Saúde da eriança e do adolescente - políticas e programas de saúde - aspectos técnicos, cientificos e éticos. Sessoes Simultaneas. In: CONGRESSO BRASILEIRO DE ENFERMAGEM, 45. Recife, 1994. Anais. Recife, ABEn. 1994. 1). 127.31 .

MNSLET-GRENN, A.: WARD PLATT. M. P. Biologia da dor e do estresse: visao geral. Clin. l'ed., v. 3. n. 3. p. 447-63. 1985.

BAIEER. C. M.: WONG. D. L. QUEST: A process of pain assessinent in children. Orthop. Nurs., v. 6, n. 1, p. 11.20. 1987.

BEYER. J.E.; WELLS. N. Avaliação da dor em crianças. Clin. Ped. Am. Norte. v. 36. n. 4, p. $881.901,1989$.

CLARO. M.T. Escala de faces para avaliagão da dor em criancas: etapa prelimiar. Ribeirão Preto, 1993. 60 p. Dissertaçao (Mestrado) - Escola de Enfermagem de Ribeirño Preto. Universidade de Så Paulo.

CLEVE. L.J.V.: SAVEDRA. M. Pain location: validity and reability of body outline markings by 4 to 7 year-old children who are hospitalized. Pediatr. Nurs.. v. 19. n. 3, p. 217.20, 1993.

CONCEIÇĀO. M.J.: SLLVA Jr.. C.A.: ROBERGE. F.X. Aspeetos do controle da dor na eriança. Rev. Bras.Anest., v. 35. n. 4. p. $311-5.1985$.

RCOFFEY. C.: DESPARMENT. J. Analgesia na cirurgia pediátrica. In: BONNET. F. et al. A dor no meio cirúrgico. Porto Alegre. Artes Médicas. 1993. cap.18. p. 278.94.

ELAND. J.M. Minimizing pain associated with prekindergarten intra-muscular injections. Issues Compr. Pediatri Nurs., v. 5. ๓. 5/6, p. 361-72. 1981 .

HESTER. N.O. The pre-operational child's reaction to imunization. Nurs. Res.. v. 28, 11. 4, p. 250.4, 1979.

Hester. N.O. Avaliação da dor aguda. Clin. Ped., v. 3, n. 3, p. 557.71 .1995$.

HURLEY, A.: WHELAN, E.G. Cognitive development and children's perception of pain. Pediatr. Nurs., v, 14, n. 1 . p. 21.4. 1988.

JERRET. M.: EVANS, K. Children's pain vocabulary. J. Adv. Nurs., v. 11. n. 4. p. 403.8. 1986.

KORCZAK. J O direito da criança ao respeito. In: DALLARI, D.A.; lioRCZAK. J. O direito da crianģa ao respeito. Sao Paulo. Summus. 1986. p. 68-99.

LE BARS. D.; WILLER. J.C. Fisiologia da sensą̧ão dolorosa. In: BONNET. F. et al. A dor no meio cirúrgico. Porto Alegre. Artes Médicas, 1993. cap.1, p. 05.47.
MACKEY, D.; JORDAN-MARSH, M. Innovative assessment of children's pain. J. Emerg.Nurs., v. 17, n. 4, p. 250-1, 1991.

MEDFORTH. N. Strategies to reduce children's perception of pain. Nurs. 'Times. v. 91. n. 2, p. 34.5, 1995.

MELZACK, R. The MeGill Pain Questionnaire: major properties and scoring methods. Pain. v. 1, p. 277.99. 1975.

MeGRATH. P.J.: CRAIG. K.D. Fatores psicológicos do desenvolvimento na dor das erianças. Clin.Ped.Am.North. v. 36, n. 4, p. 865.81, 1989.

PIAGET. J. Seis Estudos de Psicologia. Companhia Editora Forense, 146p, 1967.

PIMENTA. C. A. de M. Aspectos culturais, afetivos e terapêticos relacionados à dor no câncer. São Paulo, 1995. 132 p. Tese (Doutorado) . Escola de Enfermagem. Universidade de Sao Paulo.

PRICE. S. Pain: its experience, assessment and management in children. Nurs. Times., v. 86, 1. 9, p. 42-5, 1990.

ROSSATO, L.M.; REZENDE, M.R. A criança com dor. In: SIGAUD. C.H.S.: RAMALLO VERÍSSIMO, M.De La Ó. e org. Enfermagem pediátrica : o cuidado de enfermagem à criança e no adolescente. Săo Paulo, EPU, 1996, cap.19, p. 223-9.

SCHECHTER, N.L. Dor aguda em crianças. Clin. Ped. Am. Norte, v. 36, n. 4, p. 1109. 1989.

SCHULTZ. N.V. How ehildren perceive pain. Nurs. Outlook. v. 19, 1. 1, p. $670 \cdot 3,1971$.

SIEQEL. S. Estatistica näo paramétrica : para as ciências do comportamento. Sno Paulo, Me Graw-Hill, 1975.

STEIN. P. Indices of pain intensity: construct validity among preschoolers. Pediatr. Nurs., v. 21, n. 2, p. 119.23, 1995.

STEVENS. B.: JOHNSTON, C. Assessment and management of pain in infants. Can. Nurse. v. 88, n. 7, p. 31.4, 1992

VALLE, E.R.M. de; FRANÇOSO, L.P.C. O tratamento do câncer infantil: visão de crianças portadoras da doença. Análise de desenhos e relatos. Acta Oncol. Bras., v. 12, n. 3, p. 102-7 1992.

WONG, D.L.: BAKER, C.M. Pain in children: comparison of nssessinent scales. Pediatr. Nurs., v. 14. n. 1, p. 9-17. 1988.

YoOS. H.L. Children's illness concepts: old and new paradigms. Pediatr. Nurs., v. 20, n. 2. p. 134-40, 1994.

ZUCKERMAN, B.S.: DUBY, J.C. Abordagem evolutiva à prevença de acidentes. Clin.Ped.Am.Norte, v. 32, n. 1 , p. 15.28, 1985. 


\section{ANEXOS}

\section{ANEXO 1}

Identificação e caracterização da criança

Data da entrevista: $n^{\circ}$

Nome: Leito: - Idade:_Sexo: Data de internação:

Diagnóstico:

Tipo de procedimento que a criança foi submetida nesta internação:

Presença de familiar sim_não__ual?

\section{ANEXO 2}

Escala de Faces (CLARO 1993)
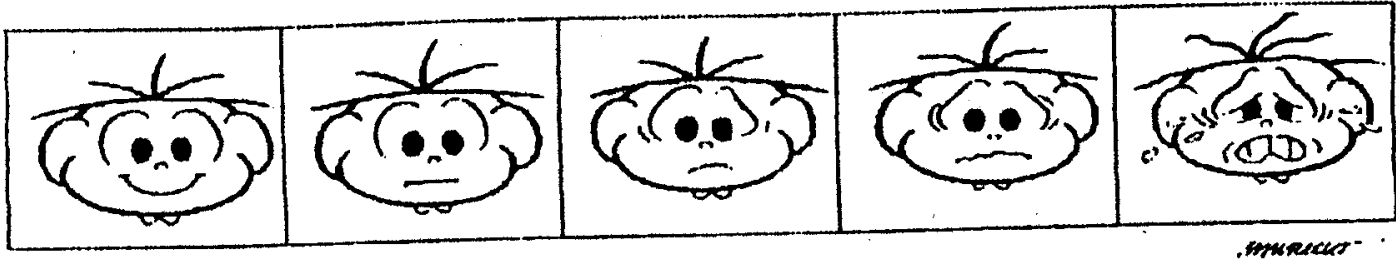

0

1

2

3

ANEXO 3

Indicadores comportamentais de dor ABU-SAAD (1984)

Vocalização

\section{Procedimento doloroso Antes Durante Depois}

- grito

- suspiro

- choro

- gemido

- soluço

Expressão Facial

- abrir a boca

- cerrar os olhos

- arregalar os olhos

- cerrar os dentes

apertar os lábios

- franzir a testa

Movimentos Corporais

- imóvel

- agitado

- protegido

- encollido

- tenso

Palavras descritoras de dor

Pergunta norteadora: "Como é a sua dor?" 